\title{
An asymptotic analysis of strategic behavior for exchange economies
}

DOI:

10.1007/s00199-017-1068-6

\section{Document Version}

Accepted author manuscript

Link to publication record in Manchester Research Explorer

\section{Citation for published version (APA):}

Koutsougeras, L. C., \& Meo, C. (2017). An asymptotic analysis of strategic behavior for exchange economies. Economic Theory, 66(0), 1-25. https://doi.org/10.1007/s00199-017-1068-6

\section{Published in:}

Economic Theory

\section{Citing this paper}

Please note that where the full-text provided on Manchester Research Explorer is the Author Accepted Manuscript or Proof version this may differ from the final Published version. If citing, it is advised that you check and use the publisher's definitive version.

\section{General rights}

Copyright and moral rights for the publications made accessible in the Research Explorer are retained by the authors and/or other copyright owners and it is a condition of accessing publications that users recognise and abide by the legal requirements associated with these rights.

\section{Takedown policy}

If you believe that this document breaches copyright please refer to the University of Manchester's Takedown Procedures [http://man.ac.uk/04Y6Bo] or contact uml.scholarlycommunications@manchester.ac.uk providing relevant details, so we can investigate your claim.

\section{OPEN ACCESS}




\title{
An Asymptotic Analysis of Strategic Behavior for Exchange Economies
}

\author{
Leonidas C. Koutsougeras • Claudia Meo
}

the date of receipt and acceptance should be inserted later

\begin{abstract}
We consider strategic market games associated to exchange economies both in a framework with no uncertainty and with asymmetric information. We address the asymptotic convergence of active Nash equilibria of strategic market games to Walrasian ones for general sequences of economies whose distribution of characteristics has compact support.
\end{abstract}

Keywords: Strategic market games; Price taking; Convergence; Asymmetric information.

JEL Classification Number: D43, D50, C72.

\section{Introduction}

Strategic market games have revealed probably the most useful model for the theory of imperfect competition. Due to the fact that they do not impose any price-taking behavior and assume that traders behave strategically, they have been successfully applied to many different areas of economics. The original model proposed by Shapley and Shubik (1977) finds applications that range from the non-cooperative foundation of competitive analysis (Mas-Colell 1982) to intertemporal models with intrinsic uncertainty (Giraud and Weyers 2004), from the analysis of bounded rationality (Voliotis 2006) to monetary models and seigniorage costs (Shubik and Tsomocos 2002).

Here we focus on the role of a large number of individuals in asymptotic results and the emergence of the price taking hypotheses. It is widely accepted among economists that in a system of markets where individual participants are small relative to the market size, individuals have a negligible effect on the determination of market outcomes, so they may be thought of as exhibiting a 'price taking' behavior. Of course, in order to make sense of this statement one has to attach a meaning to 'a small individual relative to market size'. In this way it would be possible to distinguish when price taking is a reasonable assumption and when it is not. The significance of the price taking hypothesis in economics calls for a formal clarification of this point - a 'theory of competition' so to speak.

One of the tools of economic theory to this effect is the asymptotic study of equilibrium outcomes of finite economies, when the number of individuals increases without limit. The idea is that if we can identify conditions under which equilibrium outcomes of finite economies converge asymptotically (in

The authors thank two anonymous referees for their useful comments and remarks.

L.C. Koutsougeras

School of Social Sciences, University of Manchester, Arthur Lewis 3.016, Oxford Road, Manchester M13 9PL, UK.

C. Meo (Corresponding author)

Dipartimento di Scienze Economiche e Statistiche, Università degli Studi di Napoli "Federico II", Via Cintia, 80126 Napoli, Italy.

E-mail: : claudia.meo@unina.it 
some sense) to Walrasian ones, then we would have a context where individuals have negligible effect on market outcomes and hence when 'price taking' can be justified as a reasonable hypothesis. An alternative way to view the asymptotic study is as a link between 'large finite' and 'atomless' economies. In atomless economies 'negligibility' is built in the non atomicity of the measure of the space of agents. If a large finite economy is to be thought as a reasonable substitute of the idealized continuum model, it should be the case that equilibrium outcomes of a large finite economy are close to those of the atomless limit, i.e., the equilibrium outcomes of the former should asymptotically converge (in some sense) to those of the latter as the number of individuals increases.

Asymptotic studies have been performed for a variety of equilibrium notions which have been developed in finite economies. For the most part asymptotic studies focus on the limits of the core ${ }^{1}$ and Nash equilibria, mainly because those notions are associated with the traditional theories of Edgeworth and Cournot, which are prevalent in economic theory.

In this paper we study the asymptotic limits of Nash equilibria of strategic market games both in a framework with no uncertainty and with asymmetric information. This issue has been addressed by several authors, Dubey and Shubik (1978), Mas-Colell (1982), Peck and Shell (1989), Sahi and Yao (1989), Amir et al. (1990) among many others, albeit in the fragile context of sequences of economies obtained through replication. ${ }^{2}$ Besides the particularity of this type of sequences (finite number of types of individual characteristics), the above results are shown only for 'type symmetric' Nash equilibria. It is worth remarking that in replica sequences type symmetry is a property of the core (known as 'equal treatment'), but not of Nash equilibria.

By contrast, our results apply to more general sequences of economies with characteristics drawn from compact sets and do not depend on type symmetry. One of our results provides also a rate of convergence. In this way we address the issue of asymptotic convergence of Nash equilibria, at the same level of generality as some known core convergence results. Our approach is based on the idea in Koutsougeras (2009) of measuring individuals' deviation from price taking. It is shown there that this deviation converges in proportion to zero when the number of individuals converges to infinity regardless of individual characteristics. The fact that there is some kind of convergence for arbitrary sequences is remarkable but does not fit the bill if one is specifically interested in Walrasian outcomes in the limit. In this paper we differ in two ways. First, we use a different measure of deviation from price taking which does not require differentiability of preferences. Second, the results in this paper are stronger: we demonstrate that under suitable assumptions on the distribution of individual characteristics ${ }^{3}$, the index measuring individual deviation of strategic behavior from price taking becomes arbitrarily small for each individual as the number of individuals converges to infinity, i.e., it converges to zero almost everywhere.

We extend our technique and the asymptotic convergence results to the asymmetric information environment. In such a framework the literature has proposed several alternative notions of Walrasian equilibrium. The asymptotic convergence exercise takes on an additional importance in this context, because it provides a foundation of some notion of Walrasian equilibrium.

The paper has the following structure. In Section 2 we define a market game for a model without uncertainty. Section 3 and Section 4 present the results for this model while Sections 5, 6 and 7 are devoted to the asymmetric information context. The last section concludes with final remarks.

\section{The model without uncertainty}

Let $H$ be a finite set of agents. There are $L$ commodity types in the economy and the consumption set of each agent is identified with $\Re_{+}^{L}$. Each individual $h \in H$ is characterized by a preference relation $\succeq_{h} \subset \Re_{+}^{L} \times \Re_{+}^{L}$ and an initial endowment $e_{h} \in \Re_{++}^{L}$. We use the following assumption:

\footnotetext{
1 See Anderson (1992) for a survey of core equivalence results and references.

2 In some related papers but somewhat distinct in scope, Peck and Shell (1990) features an asymptotic exercise where the number of agents remains finite but the volume of trade increases without limit, while Postlewaite and Schmeidler (1981) shows that Nash equilibria are Walrasian for a properly defined 'nearby economy'.

3 Remarkably these assumptions are the same as in the case of core convergence.
} 
Assumption 1 Preferences are continuous, strictly monotone with convex upper contours and indifference curves through the endowment do not intersect the axes.

Denote by $\mathcal{P}_{c m b}$ the set of preferences that satisfy Assumption 1 endowed with the topology of closed convergence. Let $T \subset \mathcal{P}_{c m b} \times \Re_{+}^{L}$. An economy is defined as a mapping $\mathcal{E}: H \rightarrow T$. For a given economy, the budget set of an individual at price $p \in \Re_{+}^{L}$ is $\mathcal{B}_{h}(p)=\left\{y \in \Re_{+}^{L}: p \cdot y \leq p \cdot e_{h}\right\}$.

A Walrasian equilibrium is defined as follows:

Definition 1 A Walrasian equilibrium is a pair $\left(p^{\star}, x^{\star}\right) \in \Re_{+}^{L} \times \Re_{+}^{L H}$ such that:

$$
\begin{aligned}
& \text { (i) } x_{h}^{\star} \in \mathcal{B}_{h}\left(p^{\star}\right) \text { and } \mathcal{B}_{h}\left(p^{\star}\right) \cap\left\{y \in \Re_{+}^{L}: y \succ_{h} x_{h}^{\star}\right\}=\emptyset, \forall h \in H ; \\
& \text { (ii) } \sum_{h \in H} x_{h}^{\star} \leq \sum_{h \in H} e_{h} .
\end{aligned}
$$

We now turn to describe a strategic market game, which proposes an explicit model of how exchange in the economy takes place.

\subsection{Trade using inside money}

We will develop our results for the strategic market game version appearing in Postlewaite and Schmeidler (1978) and in Peck et al. (1992) which is described below.

Trade in the economy is organized via a system of trading posts where individuals offer commodities for sale and place bids for purchases of commodities. Bids are placed in terms of a unit of account. The strategy set of each agent is $S_{h}=\left\{\left(b_{h}, q_{h}\right) \in \Re_{+}^{2 L}: q_{h}^{i} \leq e_{h}^{i}, i=1,2, \ldots, L\right\}$. Given a strategy profile $(b, q) \in \prod_{h \in H} S_{h}$, let $B^{i}=\sum_{h \in H} b_{h}^{i}$ and $Q^{i}=\sum_{h \in H} q_{h}^{i}$ denote aggregate bids and offers for each $i=1,2, \ldots, L$. Also for each agent $h$ denote $B_{-h}^{i}=\sum_{k \neq h} b_{k}^{i}, Q_{-h}^{i}=\sum_{k \neq h} q_{k}^{i}$. For a given strategy profile, the consumption of consumer $h \in H$ is determined by $x_{h}=e_{h}+z_{h}(b, q)$, where for $i=1,2, \ldots, L$ :

$$
z_{h}^{i}(b, q)= \begin{cases}\frac{b_{h}^{i}}{B^{i}} Q^{i}-q_{h}^{i} & \text { if } \sum_{i=1}^{L} \frac{B^{i}}{Q^{i}} q_{h}^{i} \geq \sum_{i=1}^{L} b_{h}^{i}, \\ -q_{h}^{i} & \text { otherwise. }\end{cases}
$$

It is postulated that whenever the term $0 / 0$ appears in the expressions above it is defined to equal zero. When $B^{i} Q^{i} \neq 0$ the fraction $\pi^{i}(b, q)=\frac{B^{i}}{Q^{i}}$ has a natural interpretation as the (average) market clearing 'price'. The relation $\sum_{i=1}^{L} \pi^{i}(b, q) q_{h}^{i} \geq \sum_{i=1}^{L} b_{h}^{i}$ is a 'bookkeeping' restriction which ensures that units of account remain at zero net supply (inside money). The interpretation of this allocation mechanism is that commodities (money) are (is) distributed among non bankrupt consumers in proportion to their bids (offers), while the purchases of bankrupt consumers are confiscated.

An equilibrium is defined as a strategy profile $(b, q) \in \prod_{h \in H} S_{h}$ that forms a Nash equilibrium in the ensuing game with strategic outcome function given by (1). Let $\mathbf{N}(\mathcal{E}) \subset \prod_{h \in H} S_{h}$ denote the set of Nash equilibrium strategy profiles of the strategic market game and $\mathcal{N}(\mathcal{E}) \subset \Re_{+}^{L H}$ the set of consumption allocations corresponding to the elements of $\mathbf{N}(\mathcal{E})$.

The following notation and familiar facts will be useful in the sequel. Fix $\left(b_{-h}, q_{-h}\right) \in \prod_{k \neq h} S_{k}$ and let $g_{h}(y)=\sum_{i=1}^{L} \frac{B_{-h}^{i}\left(y^{i}-e_{h}^{i}\right)}{Q_{-h}^{i}+e_{h}^{i}-y^{i}}{ }^{4}$. The set of allocations which an individual $h \in H$ can achieve via the strategic outcome function is given by the convex set

$$
c_{h}=\left\{y \in \Re_{+}^{L}: g_{h}(y) \leq 0, y \leq Q_{-h}+e_{h}\right\}
$$

i.e., $\left(b_{h}, q_{h}\right) \in S_{h} \Rightarrow e_{h}+z_{h}(b, q) \in c_{h}$. Conversely, $x_{h} \in c_{h} \Rightarrow \exists\left(b_{h}, q_{h}\right) \in S_{h}$ s.t. $x_{h}=e_{h}+z_{h}(b, q)$. Notice that in particular $e_{h} \in c_{h}$ because $g_{h}\left(e_{h}\right)=0$ and $e_{h} \leq Q_{-h}+e_{h}$.

${ }^{4}$ In order to save on notation we omit the dependency on $\left(b_{-h}, q_{-h}\right)$. In the results the values of those variables will be fixed so no confusion should arise. 
At an equilibrium with nonzero bids and offers we have that $\bar{x} \in \mathcal{N}(\mathcal{E})$ if and only if:

$$
\begin{aligned}
& \text { (i) } \bar{x}=x(\bar{b}, \bar{q}) \text {, for some }(\bar{b}, \bar{q}) \in \prod_{h \in H} S_{h} \text {; } \\
& \text { (ii) } \forall h \in H, c_{h} \cap\left\{y \in \Re_{+}^{L}: y \succ_{h} \bar{x}_{h}\right\}=\emptyset .
\end{aligned}
$$

We say that $\bar{x} \in \mathcal{N}(\mathcal{E})$ is fully active if for the corresponding $(\bar{b}, \bar{q}) \in \mathbf{N}(\mathcal{E})$ we have $\pi(\bar{b}, \bar{q}) \gg 0$, i.e., there is activity in all trading posts. In the sequel we will focus on such equilibria ${ }^{5}$. The existence of such equilibria is established in several articles, notably in Mas-Colell (1982) or Peck and Shell (1992).

\subsection{Strategic vs price taking behavior}

Let us fix a fully active $\bar{x} \in \mathcal{N}(\mathcal{E})$ corresponding to a strategy profile $(\bar{b}, \bar{q}) \in \mathbf{N}(\mathcal{E})$. Consider one $h \in H$ and denote $\bar{z}_{h}=\bar{x}_{h}-e_{h}$.

The monotonicity of preferences implies that $g_{h}\left(\bar{x}_{h}\right)=0$, i.e., $\bar{x}_{h}$ lies on the boundary of the convex set $c_{h}$, which is $C^{2}$. Since the sets of preferable bundles are also convex, by the separating hyperplane theorem there is a $p_{h} \in \Re_{+}^{L}$, specifically $p_{h}=D g_{h}\left(\bar{x}_{h}\right)$, where $D g_{h}(\cdot)$ denotes the gradient of $g_{h}(\cdot)$, such that:

$$
w \succeq_{h} \bar{x}_{h} \Rightarrow p_{h} \cdot w \geq p_{h} \cdot \bar{x}_{h} \text { and } w \in c_{h} \Rightarrow p_{h} \cdot w \leq p_{h} \cdot \bar{x}_{h} .
$$

Using the definition of $c_{h}$ we have:

$$
p_{h}=D g_{h}\left(\bar{x}_{h}\right)=\left(\frac{\bar{B}_{-h}^{i} \bar{Q}_{-h}^{i}}{\left(\bar{Q}_{-h}^{i}-\bar{z}_{h}^{i}\right)^{2}}\right)_{i=1}^{L}=\left(\pi^{i}(\bar{b}, \bar{q}) \frac{\bar{Q}_{-h}^{i}}{\left(\bar{Q}_{-h}^{i}-\bar{z}_{h}^{i}\right)}\right)_{i=1}^{L} .
$$

Now observe that if for some $\lambda_{h}>0, p_{h}=\lambda_{h} \pi(\bar{b}, \bar{q})$ then the behavior of such an individual would be identical to price taking at the market clearing prices $\pi(\bar{b}, \bar{q})$. To see this notice that: (a) $\pi(\bar{b}, \bar{q}) \bar{x}_{h}=$ $\pi(\bar{b}, \bar{q}) e_{h}$ and (b) since $\pi(\bar{b}, \bar{q}) \gg 0\left(\bar{x}\right.$ is active), there is a cheaper point, i.e., $w \in \Re_{+}^{L}$ with $\pi(\bar{b}, \bar{q}) w<$ $\pi(\bar{b}, \bar{q}) \bar{x}_{h}=\pi(\bar{b}, \bar{q}) e_{h}$. Since preferences are continuous and upper contours convex, the first part of (4) implies $y \succ_{h} \bar{x}_{h} \Rightarrow \pi(\bar{b}, \bar{q}) y>\pi(\bar{b}, \bar{q}) e_{h}$.

Therefore, the measurement

$$
\delta_{h}(\bar{x})=\max \left\{\left|\frac{p_{h}^{i}}{p_{h}^{j}} \cdot \frac{\pi^{j}(\bar{b}, \bar{q})}{\pi^{i}(\bar{b}, \bar{q})}-1\right|: i, j=1,2, \ldots, L\right\}
$$

serves as an indicator of 'how far' the strategic behavior of individual $h$ falls from price taking ${ }^{6}$. When $\delta_{h}(\bar{x}) \neq 0$, the slope of the attainable allocations $\left(\frac{p_{h}^{i}}{p_{h}^{j}}\right)$ at $\bar{x}$ differs from the slope of the budget defined by the market clearing prices $\left(\frac{\pi^{i}(\bar{b}, \bar{q})}{\pi^{j}(b, \bar{q})}\right)$. In other words, the individual can attain bundles near $\bar{x}$, which are not budget feasible at the market clearing prices. Since attainable allocations are always budget feasible at corresponding market clearing prices we conclude that any move to attainable bundles nearby $\bar{x}$ alter market clearing prices, i.e., the actions of the individual affects market prices.

Clearly, for each agent $h$ we have $\delta_{h}(\bar{x}) \geq 0$ and $\bar{x}$ is Walrasian if (and only if) $\delta_{h}(\bar{x})=0$ for each agent $h$. Therefore, a sequence of market game price-allocation pairs tends to become a price taking one, if (and only if) the above indicator tends to zero (in an appropriate sense) for all individuals.

We are ready now to proceed with the results of this paper.

\footnotetext{
5 Alternatively, we could consider the subset of commodities $L^{\prime}$ for which there is active trade.

${ }^{6}$ In the case of $C^{2}$ preferences, the indicator $\delta_{h}(\cdot)$ coincides with $\gamma_{h}(\cdot)$ in Koutsougeras (2009).
} 


\section{Results for economies with no uncertainty}

For the results that follow we consider a sequence $\left\{\mathcal{E}_{n}\right\}_{n \in N}$ of economies $\mathcal{E}_{n}: H_{n} \rightarrow \mathcal{P}_{c m b} \times\left[\frac{1}{r}, r\right]^{L}$, where $\# H_{n} \rightarrow \infty, \lim \frac{1}{\# H_{n}} \sum_{h \in H_{n}} e_{h} \gg 0$ and associated $x_{n} \in \mathcal{N}\left(\mathcal{E}_{n}\right)$, for each $n \in N$ which are fully active. Let $\left(b_{n}, q_{n}\right) \in \mathbf{N}\left(\mathcal{E}_{n}\right)$ be the corresponding strategies.

The following result is shown in Koutsougeras (2009) and its proof applies unchanged here.

Theorem 1 For each $\epsilon>0$, there is an $n_{\epsilon} \in N$ so that for all $n>n_{\epsilon}$

$$
\frac{1}{\# H_{n}} \#\left\{h \in H_{n}: \delta_{h}\left(x_{n}\right)>\epsilon\right\}<\epsilon
$$

or, equivalently:

$$
\frac{1}{\# H_{n}} \#\left\{h \in H_{n}: \delta_{h}\left(x_{n}\right)>\epsilon\right\} \rightarrow 0
$$

The above theorem asserts some kind of convergence (in measure) for the indicator $\delta_{h}$. Its strength is that it requires no assumptions, so it applies to all sequences of active Nash equilibria. On the other hand it hardly fits the bill: we still need to show some convergence of the Nash equilibrium allocations themselves. Furthermore, we need to ensure that 'most' of the commodities are consumed by 'most' of the individuals who exhibit 'almost' a price taking behavior, as the above theorem asserts. We proceed with two lemmas which will be useful to us in pursuing this end.

Lemma $1 \frac{1}{\# H_{n}} \#\left\{h \in H_{n}: x_{n, h} \leq k r 1_{L}\right\}>1-\frac{L}{k}$, for each $k>L$.

Proof.

Define $T_{n}^{i}(k)=\left\{h \in H_{n}: x_{n, h}^{i}>k r\right\}$, for $i=1,2, \ldots, L$. Notice that $\#\left\{h \in H_{n}: x_{n, h} \leq k r 1_{L}\right\}=\# H_{n}-$ \# $\left(\bigcup_{i=1}^{L} T_{n}^{i}(k)\right)$.

From the definition of $T_{n}^{i}(k)$ it follows that:

$$
\begin{aligned}
\# T_{n}^{i}(k) \cdot k \cdot r & <\sum_{h \in T_{n}^{i}(k)} x_{n, h}^{i}= \\
& =\sum_{h \in T_{n}^{i}(k)} e_{n, h}^{i}+Q_{n}^{i} \cdot \sum_{h \in T_{n}^{i}(k)} \frac{b_{n, h}^{i}}{B_{n}^{i}}-\sum_{h \in T_{n}^{i}(k)} q_{n, h}^{i} \leq \\
& \leq \sum_{h \in T_{n}^{i}(k)} e_{n, h}^{i}+Q_{n}^{i}-\sum_{h \in T_{n}^{i}(k)} q_{n, h}^{i}= \\
& =\sum_{h \in T_{n}^{i}(k)} e_{n, h}^{i}+\sum_{h \notin T_{n}^{i}(k)} q_{n, h}^{i} \leq \\
& \leq \# T_{n}^{i}(k) \cdot r+\left(\# H_{n}-\# T_{n}^{i}(k)\right) \cdot r .
\end{aligned}
$$

Therefore, we conclude that $\# T_{n}^{i}(k)<\# H_{n} \cdot \frac{1}{k}$.

It then follows that:

$$
\#\left(\bigcup_{i=1}^{L} T_{n}^{i}(k)\right) \leq \sum_{i=1}^{L} T_{n}^{i}(k)<\# H_{n} \cdot \frac{L}{k}
$$

Hence:

$$
\#\left\{h \in H_{n}: x_{n, h} \leq k r 1_{L}\right\}=\# H_{n}-\#\left(\bigcup_{i=1}^{L} T_{n}^{i}(k)\right)>\# H_{n} \cdot\left(1-\frac{L}{k}\right)
$$

Consider now a sequence of economies $\mathcal{E}_{n}: H_{n} \rightarrow T$ where $T \subset \mathcal{P}_{c m b} \times\left[\frac{1}{r}, r\right]^{L}$ is compact. 
Lemma 2 For each $i=1,2, \ldots, L$, there is a subsequence (still indexed by $n$ ), and $\epsilon>0$ so that: $\#\left\{h \in H_{n}: x_{n, h}^{i} \geq \epsilon\right\} \geq \# H_{n} \epsilon$ for $n$ sufficiently large.

Proof.

Step I

Fix a commodity $i=1,2, \ldots, L$. From the above lemma for $k=2 L$ we have:

$$
\begin{gathered}
\frac{1}{\# H_{m}} \# \bigcap_{n=1}^{m} \bigcup_{k=n}^{m}\left\{h \in H_{k}: x_{k, h} \leq 2 L r 1_{L}\right\}= \\
\quad=\frac{1}{\# H_{m}} \#\left\{h \in H_{m}: x_{m, h} \leq 2 L r 1_{L}\right\}>\frac{1}{2} .
\end{gathered}
$$

Therefore, $\lim _{m \rightarrow \infty} \frac{1}{\# H_{m}} \# \bigcap_{n=1}^{m} \bigcup_{k=n}^{m}\left\{h \in H_{k}: x_{k, h} \leq 2 L r 1_{L}\right\} \geq \frac{1}{2}$.

Consider then any $t \in \bigcap_{n=1}^{\infty} \bigcup_{k=n}^{\infty}\left\{h \in H_{k}: x_{k, h} \leq 2 L r 1_{L}\right\}$. We may assume by passing to a subsequence if necessary, that $\left(\succeq_{n, t}, e_{n, t}\right) \rightarrow\left(\succeq_{t}, e_{t}\right) \in T$ and $x_{n, t} \rightarrow x_{t} \leq 2 L r 1_{L}$. Since $x_{n, t} \succeq_{n, t} e_{n, t}$, we have that $x_{t} \succeq_{t} e_{t} \geq \frac{1}{r} 1_{L}$, which implies that $x_{t} \gg 0$. In particular, we conclude that $x_{t}^{i}>0$ for each $t \in \bigcap_{n=1}^{\infty} \bigcup_{k=n}^{\infty}\left\{h \in H_{k}: x_{k, h} \leq 2 L r\right\}$.

Step II

Suppose the claim of the lemma is false. Then for each $\epsilon>0$ and $N$, there is $n \geq N$ so that $\frac{1}{\# H_{n}} \#\{h \in$ $\left.H_{n}: x_{n, h}^{i} \geq \epsilon\right\}<\epsilon$.

By choosing a sequence $\epsilon_{n} \rightarrow 0$ and $N \rightarrow \infty$ and passing to a subsequence (still indexed by $n$ ) we have that:

$$
\frac{1}{\# H_{n}} \#\left\{h \in H_{n}: x_{n, h}^{i} \geq \epsilon_{n}\right\}<\epsilon_{n}
$$

It follows that:

$$
\begin{aligned}
\frac{1}{\# H_{m}} \# \bigcap_{n=1}^{m} \bigcup_{k=n}^{m}\left\{h \in H_{k}: x_{k, h}^{i} \geq \epsilon_{k}\right\} & \leq \frac{1}{\# H_{m}} \#\left\{h \in H_{m}: x_{m, h}^{i} \geq \epsilon_{m}\right\}< \\
& <\epsilon_{m} .
\end{aligned}
$$

We conclude that $\lim _{m \rightarrow \infty} \frac{1}{\# H_{m}} \# \bigcap_{n=1}^{m} \bigcup_{k=n}^{m}\left\{h \in H_{k}: x_{k, h}^{i} \geq \epsilon_{k}\right\}=0$, which implies that for almost all $t \in \bigcap_{n=1}^{\infty} \bigcup_{k=n}^{\infty}\left\{h \in H_{k}: x_{k, h} \leq 2 L r\right\}$ we have $\lim _{n \rightarrow \infty} x_{n, t}^{i}=0$.

Therefore, $x_{t}^{i}=0$ for almost all $t \in \bigcap_{n=1}^{\infty} \bigcup_{k=n}^{\infty}\left\{h \in H_{k}: x_{k, h} \leq 2 L r\right\}$, which contradicts the conclusion of Step I. This contradiction establishes the claim of the lemma.

We now turn to develop an asymptotic convergence theorem, by introducing appropriate assumptions on the distribution of characteristics along a sequence of economies. In particular, consider a sequence of economies $\mathcal{E}_{n}: H_{n} \rightarrow T$ where $T \subset \mathcal{P}_{c m b} \times\left[\frac{1}{r}, r\right]^{L}$ is compact. For such sequences the set of Nash equilibrium allocations is uniformly bounded as the following result shows.

Proposition 1 Let $\left\{\mathcal{E}_{n}\right\}_{n \in N}$ be a sequence of economies, $\mathcal{E}_{n}: H_{n} \rightarrow T$ where $\# H_{n} \rightarrow \infty$ and let $x_{n} \in \mathcal{N}\left(\mathcal{E}_{n}\right)$, for each $n \in N$ be fully active. There is $B \subset \Re_{+}^{L}$, which is bounded and depends only on $T$, such that for all $n \in N x_{n, h} \in B$ for each $h \in H_{n}$, i.e., the set of Nash equilibrium allocations remains uniformly bounded along a sequence of economies with characteristics drawn from $T$.

Proof.

Step I. Let $\pi_{n}=\pi\left(b_{n}, q_{n}\right)$ and normalize prices so that $\sum_{i=1}^{L} \pi_{n}^{i}=1$.

Suppose that $\sup \left\{x_{n, h}^{j}: h \in H_{n}\right\} \rightarrow \infty$ for some $j=1,2, \ldots, L$. Then it must be $\sup \left\{\frac{b_{n, h}^{j}}{\pi_{n}^{j}}-q_{n, h}^{j}: h \in\right.$ $\left.H_{n}\right\} \rightarrow \infty$. It follows that $\pi_{n}^{j} \rightarrow 0$. Hence, there must be $\pi_{n}^{i}>1 / L$ for some $i \neq j$ along a subsequence, so $\pi_{n}^{i} / \pi_{n}^{j} \rightarrow \infty$. 
Step II. By Lemma 2, passing to a subsequence if necessary, we may assume that for some $1>\epsilon>0$,

$$
\#\left\{h \in H_{n}: x_{n, h}^{i} \geq \epsilon\right\} \geq \# H_{n} \epsilon .
$$

Also by Lemma 1 , setting $k \geq 2 L \epsilon^{-1}-1$, we have that for all $n \in N$,

$$
\#\left\{h \in H_{n}:\left|z_{n, h}^{i}\right| \leq\left(2 L \epsilon^{-1}-1\right) r \forall i=1,2, \ldots, L\right\}>\# H_{n}\left(1-\frac{\epsilon}{2}\right) .
$$

Step III. We now show the following claim: for some subsequence (still indexed by $n$ ) there exists $M>0$ so that:

$$
\#\left\{h \in H_{n}: \frac{p_{h}^{i}}{p_{h}^{j}} \leq M\right\}>\# H_{n} \frac{\epsilon}{2} .
$$

Suppose not. Then for every $M>0$ we have $\#\left\{h \in H_{n}: \frac{p_{h}^{i}}{p_{h}^{j}} \leq M\right\} \leq \# H_{n} \frac{\epsilon}{2}$ or equivalently $\#\left\{h \in H_{n}: \frac{p_{h}^{i}}{p_{h}^{j}}>M\right\}>\# H_{n}\left(1-\frac{\epsilon}{2}\right)$. In conclusion, we have for every $M>0$ :

$$
\#\left\{h \in H_{n}: \frac{p_{h}^{j}}{p_{h}^{i}}<M^{-1}\right\}>\# H_{n}\left(1-\frac{\epsilon}{2}\right)
$$

In this case, (10) along with (7) and (8), implies that for each $n \in N$ there is $h_{n} \in H_{n}$ so that the following is true: $\left|z_{h_{n}}\right| \leq\left(2 L \epsilon^{-1}-1\right) r \cdot 1_{L}$, so that along some subsequence (still indexed by $\left.n\right) z_{h_{n}} \rightarrow z$, $z_{h_{n}}^{i}+e_{h_{n}}^{i} \geq \epsilon$ and $\frac{p_{h_{n}}^{j}}{p_{h_{n}}^{i}} \rightarrow 0$.

The compactness of $T$ implies that, by passing to a subsequence if necessary we may assume that $\left(\succeq_{h_{n}}, e_{h_{n}}\right) \rightarrow(\succeq, e) \in T$.

Consider for each $n \in N$ the vectors $t_{n} \in \Re_{+}^{L}$ where $t_{n}^{i}=-\frac{p_{h_{n}}^{j}}{p_{h_{n}}^{i}}, t_{n}^{j}=1$ and $t_{n}^{l}=0$ for $l \neq i, j$. For these vectors we have that $p_{h_{n}} t_{n}=0,\left|t_{n}^{i}\right|<\epsilon$ for $n$ large enough, $t_{n} \rightarrow t \geq 0$ and $t \neq 0$. By the convexity of preferences it must be that $z_{h_{n}}+e_{h_{n}} \succeq_{h_{n}} z_{h_{n}}+e_{h_{n}}+t_{n}$. Taking limits we conclude that $z+e \succeq z+e+t$ which contradicts the monotonicity of $\succeq$. This contradiction establishes our claim that (9) is true for some $M>0$. So in this step we can conclude that there exists $M>0$ so that:

$$
\frac{1}{\# H_{n}} \#\left\{h \in H_{n}: \frac{p_{h}^{i}}{p_{h}^{j}}>M\right\}<1-\frac{\epsilon}{2} .
$$

Step IV. Since $\pi_{n}^{i} / \pi_{n}^{j} \rightarrow \infty$ we have that $\pi_{n}^{i} / \pi_{n}^{j}(1-\epsilon)>M$ for $n$ large enough. Furthermore, by Theorem 1 we have that for $n$ large enough:

$$
\frac{1}{\# H_{n}} \#\left\{h \in H_{n}: \delta_{h}\left(x_{n}\right) \leq \epsilon\right\} \geq 1-\frac{\epsilon}{2} .
$$

But then for $n$ large enough we have the following string of inequalities:

$$
\begin{aligned}
\frac{1}{\# H_{n}} \#\left\{h \in H_{n}: \frac{p_{h}^{i}}{p_{h}^{j}}>M\right\} & \geq \frac{1}{\# H_{n}} \#\left\{h \in H_{n}: \frac{p_{h}^{i}}{p_{h}^{j}} \geq \frac{\pi_{n}^{i}}{\pi_{n}^{j}}(1-\epsilon)\right\} \\
& \geq \frac{1}{\# H_{n}} \#\left\{h \in H_{n}: \delta_{h}\left(x_{n}\right) \leq \epsilon\right\} \\
& \geq 1-\frac{\epsilon}{2},
\end{aligned}
$$

which contradicts (11).

We can now prove the following result. 
Theorem 2 Consider a sequence of economies $\left\{\mathcal{E}_{n}\right\}_{n \in N}$, where $\mathcal{E}_{n}: H_{n} \rightarrow T, \# H_{n} \rightarrow \infty$ and $T \subset$ $\mathcal{P}_{c m b} \times\left[\frac{1}{r}, r\right]^{L}$ is compact. Let $\left(b_{n}, q_{n}\right) \in \mathbf{N}\left(\mathcal{E}_{n}\right)$ and suppose that for some $\beta>0, \frac{1}{\# H_{n}} Q_{n} \gg \beta 1_{L}$ for $n$ large enough, so that the corresponding $x_{n} \in \mathcal{N}\left(\mathcal{E}_{n}\right)$ is fully active. Then, given any $\epsilon>0$ there is $N$ so that if $\# H_{n}>N$, then $\delta_{h}\left(x_{n}\right)<\epsilon, \forall h \in H_{n}$.

Proof.

Since $T$ is compact, by Proposition 1 we have that for each $h \in H_{n},\left\|z_{n, h}\right\|=\left\|x_{n, h}-e_{h}\right\| \leq c 1_{L}$ for some $c>0$.

Furthermore, since for $n$ large enough $\frac{1}{\# H_{n}} Q_{n} \gg \beta 1_{L}$, by passing to a subsequence if necessary we may assume that there is $\xi>0$ so that $\#\left\{h \in H_{n}: q_{h}^{i} \geq \xi\right\} \geq \# H_{n} \xi$ for all $i=1,2, \ldots, L$. In this case, when $n$ is large enough, we have that for all $h \in H_{n}$ :

$$
Q_{n,-h}^{i}=Q_{n}^{i}-q_{n, h}^{i} \geq Q_{n}^{i}-r \geq \# H_{n} \xi^{2}-r .
$$

Fix one $h \in H_{n}$. We have $\frac{z_{n, h}^{i}}{Q_{n,-h}^{i}} \leq \frac{c}{\# H_{n} \xi^{2}-r}$ for all $i=1,2, \ldots, L$. It follows that:

$$
\begin{aligned}
\left|\frac{p_{h}^{i}}{p_{h}^{j}} \cdot \frac{\pi^{j}(\bar{b}, \bar{q})}{\pi^{i}(\bar{b}, \bar{q})}-1\right| & =\left|\frac{Q_{n,-h}^{i}\left(Q_{n,-h}^{j}-z_{n, h}^{j}\right)}{Q_{n,-h}^{j}\left(Q_{n,-h}^{i}-z_{n, h}^{i}\right)}-1\right| \\
& =\left|\frac{\frac{z_{n, h}^{i}}{Q_{n,-h}^{i}}}{1-\frac{z_{n, h}^{i}}{Q_{n,-h}^{i}}}-\frac{\frac{z_{n, h}^{j}}{Q_{n,-h}^{j}}}{1-\frac{z_{n, h}^{i}}{Q_{n,-h}^{i}}}\right| \\
& \leq 2 \frac{\frac{c}{\# H_{n} \xi^{2}-r}}{1-\frac{c}{\# H_{n} \xi^{2}-r}}=2 \frac{c}{\# H_{n} \xi^{2}-r-c}
\end{aligned}
$$

Hence, $\delta_{h}\left(x_{n}\right)=\max \left\{\left|\frac{p_{h}^{i}}{p_{h}^{j}} \cdot \frac{\pi_{n}^{j}}{\pi_{n}^{i}}-1\right|: i, j=1,2, \ldots, L\right\} \leq 2 \frac{c}{\# H_{n} \xi^{2}-r-c}$. Therefore, given $\epsilon>0$ by choosing $N=\frac{2 c+\epsilon(r+c)}{\epsilon \xi^{2}}$ we have that if $\# H_{n}>N$ then $\delta_{h}\left(x_{n}\right)<\epsilon$ for every $h \in H_{n}$, as desired.

\section{Purely competitive sequences of economies with no uncertainty}

The results of the previous section can become more transparent by considering sequences of economies converging to a limit. To this end in this section we will consider 'purely competitive' sequences of economies (see Hildenbrand (1974), p. 138) which are defined as follows.

Let $T \subset \mathcal{P}_{c m b} \times \Re_{+}^{L}$ be compact. Consider a sequence $\left\{\mathcal{E}_{n}\right\}_{n \in N}$, where $\mathcal{E}_{n}: H_{n} \rightarrow T$ such that:

(i) $\# H_{n} \rightarrow \infty$.

(ii) The sequence of distributions of characteristics $\left(\mu_{n}\right)$ converges weakly on $T$.

(iii) If $\mu=\lim \mu_{n}$ then $\int e d \mu_{n} \rightarrow \int e d \mu$.

(iv) $\int e d \mu>0$.

Let $\left\{\mathcal{E}_{n}\right\}_{n \in N}$ be such a sequence and consider a sequence of fully active Nash equilibria $x_{n} \in \mathcal{N}\left(\mathcal{E}_{n}\right)$. Since $T$ is compact, it follows by Proposition 1 that $\left\{x_{n}\right\}_{n \in N}$ is uniformly bounded, so we can extract a subsequence (still indexed by $n$ ) which converges in distribution, i.e., by defining for each $B \in \Re^{L}$ $\lambda_{n}(B)=\frac{1}{\# H_{n}} \#\left\{h \in H_{n}: x_{n, h} \in B\right\}$ we have that $\lambda_{n} \rightarrow \lambda$ weakly.

Denote now by $\tau_{n}$ the joint distribution of $\left(\mathcal{E}_{n}, x_{n}\right): H_{n} \rightarrow T \times \Re^{L}$. The sequence $\left(\tau_{n}\right)_{n \in N}$ is tight since the sequences of its marginal distributions are tight, so we may assume, by passing to a subsequence if necessary, that $\tau_{n} \rightarrow \tau$ weakly. Hence, this sequence of economies and associated allocations admits a continuous representation (see Hildenbrand (1974), Proposition 2, p. 139): there is an atomless measure 
space $(H, \mathcal{H}, \nu),(\mathcal{E}, x): H \rightarrow T \times \Re^{L}$ and measurable functions $a_{n}: H \rightarrow H_{n}$, so that $\left(\mathcal{E}_{n}\left(a_{n}\right), x_{n, a_{n}}\right) \rightarrow$ $(\mathcal{E}, x)$ a.e. in $H$ and the respective distributions of $\left(\mathcal{E}_{n}\left(a_{n}\right), x_{n, a_{n}}\right)$ and $(\mathcal{E}, x)$ are $\tau_{n}$ and $\tau$ respectively.

Using this continuous representation, our indicator can be extended in a natural way on $H$, by $\hat{\delta}_{h}\left(x_{n, a_{n}}\right)=\delta_{a_{n}(h)}\left(x_{n}\right)$. The meaning of Theorem 1 can be made more transparent as follows:

Lemma $3 \hat{\delta}_{h}\left(x_{n, a_{n}}\right) \rightarrow 0$ in measure.

Proof.

By definition of a continuous representation of the sequence of economies:

$$
\begin{aligned}
\nu\left(\left\{h \in H: \hat{\delta}_{h}\left(x_{n, a_{n}}\right)>\epsilon\right\}\right) & =\nu\left(\left\{h \in H: \delta_{a_{n}(h)}\left(x_{n, a_{n}}\right)>\epsilon\right\}\right) \\
& =\nu\left(a_{n}^{-1}\left(\left\{h \in H_{n}: \delta_{h}\left(x_{n, a_{n}}\right)>\epsilon\right\}\right)\right) \\
& =\frac{1}{\# H_{n}} \#\left\{h \in H_{n}: \delta_{h}\left(x_{n}\right)>\epsilon\right\} .
\end{aligned}
$$

By Theorem 1 the righthand side converges to zero.

The following proposition establishes that the allocation $x$ is Walrasian for the economy $\mathcal{E}$, provided that the associated sequence of strategic prices does not converge to the boundary of $\Re_{+}^{L}$.

Proposition 2 Let $x_{n}$ be fully active for each $n \in N$ and suppose that the sequence of associated strategic market game prices $\left\{\pi_{n}\right\}_{n \in N}$ are such that no subsequence converges to the boundary of $\Re_{+}^{L}$. Then $\delta_{h}(x)=$ 0, a.e. in $H$.

Proof.

Normalizing prices so that $\sum_{i=1}^{L} \pi_{n}^{i}=1$, for each $n \in N$, we may assume, by passing to a subsequence if necessary, that $\pi_{n} \rightarrow p>0$. For $i=1,2, \ldots, L$ let $b_{h}^{i}=p^{i} x_{h}^{i}$ and $q_{h}^{i}=e_{h}^{i}$ a.e. in $H$. It can be verified that $\pi(b, q)=p$ and $e_{h}+z_{h}(b, q)=x_{h}$. Thus, $\delta_{h}(x)$ is well defined and since $\left(\mathcal{E}_{n}\left(a_{n}\right), x_{n, a_{n}}\right) \rightarrow(\mathcal{E}, x)$ a.e. in $H$, it follows by continuity of $\hat{\delta}_{h}(\cdot)$ that $\delta_{h}(x)=\lim \hat{\delta}_{h}\left(x_{n, a_{n}}\right)$, a.e. in $H$. By lemma 3 above, $\hat{\delta}_{h}\left(x_{n, a_{n}}\right) \rightarrow 0$ in measure so there is a subsequence $\hat{\delta}_{h}\left(x_{n_{k}, a_{n_{k}}}\right) \rightarrow 0$ a.e. in $H$. Since $\delta_{h}(x)=\lim \hat{\delta}_{h}\left(x_{n_{k}, a_{n_{k}}}\right)$, a.e. in $H$, it follows that it must be $\delta_{h}(x)=0$, a.e. in $H$.

\section{The model with uncertainty and asymmetric information}

\subsection{Asymmetric information economies}

We introduce now some exogenous uncertainty and asymmetries in the information possessed by traders in the environment described before, that is, an economy with a finite population of agents, indexed by $h \in H=\{1, \ldots, m\}$, and a finite number $L$ of commodities $i \in\{1, \ldots, L\}$.

The exogenous uncertainty is formulated in terms of a measurable space $(\Omega, \mathcal{F})$, with $\Omega$ denoting a finite set of $k$ distinct states of nature and the field $\mathcal{F}$ representing the set of all the events. The information initially possessed by trader $h$ is modelled by a measurable partition $\Pi_{h}$ of $\Omega$. The asymmetries in the initial information refer to the fact that partitions differ across traders. Agents make contracts which may be contingent on the state of nature that will materialize in the future: that is, we assume that trading takes place by means of ex ante contractual arrangements. Once uncertainty is resolved, each trader receives his private information: after receiving his private information, he is not necessarily able to distinguish which state of nature $s \in \Omega$ actually occurs but he can just observe (and, if necessary, prove in front of a court of law) the element $\Pi_{h}(s)$ of his partition $\Pi_{h}$ that contains such state. In this period he receives his endowment of physical resources and agreements are carried out.

$\Re^{L}$ is the commodity space and the consumption set of each agent in every state of nature is identified with 
$\Re_{+}^{L}$. Each trader $h \in H$ is described by three primitives: his initial information, described by a partition $\Pi_{h}$ of $\Omega$; his state-dependent initial endowment of physical resources given by a function $e_{h}: \Omega \longrightarrow \Re_{++}^{L}$ or, equivalently, by a vector $e_{h} \in \Re_{++}^{L k}$; his preferences over contingent consumption plans $x_{h} \in \Re^{L k}$ represented by a utility function $u_{h}: \Re_{+}^{L k} \rightarrow \Re$. We impose on utility functions the same assumptions as in the deterministic framework.

ASSUMPTION 2 Utility functions are continuous, concave, strictly monotone and indifference curves through the endowment do not intersect the axes.

We denote by $\mathcal{U}_{c}$ the set of utility functions that satisfy Assumption 2.

Given a partition $\Pi$ of $\Omega$, a commodity bundle $x=(x(s))_{s \in \Omega} \in \Re_{+}^{L k}$ is said to be $\Pi$-measurable when it is constant over the elements of the partition $\Pi$.

The assumption that $e_{h}$ is $\Pi_{h}$-measurable is standard (see, for example, Yannelis (1991)). If the endowments were not such a way, then an agent could use the fact that his endowment varies over states which he cannot distinguish to refine information.

An exchange economy with asymmetric information is thus described by a mapping $\mathcal{E}: H \rightarrow \operatorname{Part}(\Omega) \times$ $\Re_{+}^{L \cdot k} \times \mathcal{U}_{c}$ where $\operatorname{Part}(\Omega)$ denotes the set of all the partitions of $\Omega$. We assume that traders share common knowledge as to the partitions $\Pi_{h}$.

A price system is a vector $p=(p(s))_{s \in \Omega}$ that specifies a commodity price $p(s) \in \Re_{+}^{L}$ in each state $s \in \Omega$. For a given economy with asymmetric information, a Radner equilibrium is defined as follows.

Definition 2 A Radner equilibrium is a pair $\left(p^{\star}, x^{\star}\right) \in \Re_{+}^{L k} \times \Re_{+}^{L k H}$ such that:

(i) $x_{h}^{\star}$ is $\Pi_{h}-$ measurable, $\forall h \in H$;

(ii) $x_{h}^{\star} \in \arg \max \left\{u_{h}(y): p^{\star} \cdot y \leq p^{\star} \cdot e_{h}, y\right.$ is $\Pi_{h}-$ measurable $\}, \forall h \in H$;

(iii) $\sum_{h \in H} x_{h}^{\star} \leq \sum_{h \in H} e_{h}$.

We now turn to describe a strategic market game, which proposes an explicit model of how exchange in the economy takes place.

5.2 The trading process with inside money

The trading process in this economy runs as follows. In every state of nature there is a trading post for each commodity to which individuals bring commodities for sale and place bids for purchases of commodities. Let $q_{h}^{i}(s)$ be the offer of commodity $i$ by consumer $h$ in state $s$ and $b_{h}^{i}(s)$ the bid for commodity $i$ by consumer $h$ in state $s$.

Since offers must be in terms of physical commodities, it must hold true for every state $s \in \Omega$ that

$$
q_{h}^{i}(s) \leq e_{h}^{i}(s) .
$$

Individuals choose strategies that specify bids and offers for each commodity in every state of nature; hence, the strategy set of each agent $h$ is:

$$
S_{h}=\left\{\left(b_{h}, q_{h}\right) \in \Re_{+}^{2 L k}: q_{h}^{i}(s) \leq e_{h}^{i}(s), \forall i \in\{1, \ldots, L\} \text { and } \forall s \in \Omega\right\} .
$$

It is worth noting that traders are not restricted to choose the same bids and offers in states of nature that they cannot distinguish; that is, we do not impose any measurability constraint on the strategies of the consumers with respect to their information sets. Also Fugarolas et al. (2009) associate a game to the asymmetric information economies where the players'strategies are not required to be measurable with respect to their initial information. However, their aim is different from ours: they recover Walrasian expectations equilibria of the economy as Nash equilibria of the associated game.

Following the same line as before, capital letters denote aggregate quantities; precisely, given a strategy profile $(b, q) \in \prod_{h \in H} S_{h}$ and a state $s \in \Omega$, let $B^{i}(s)=\sum_{h \in H} b_{h}^{i}(s)$ and $Q^{i}(s)=\sum_{h \in H} q_{h}^{i}(s)$ denote 
aggregate bids and offers in the state of nature $s$ for each $i=1,2, \ldots, L$. Also, the subscript $-h$ has to be interpreted, as usual, as 'all individuals except $h$ '. Hence, for each agent $h$ denote $B_{-h}^{i}(s)=\sum_{k \neq h} b_{k}^{i}(s)$ and $Q_{-h}^{i}=\sum_{k \neq h} q_{k}^{i}(s)$.

Similarly to the deterministic case, trade proceeds as follows. Each trader faces a unique budget constraint across states of nature expressed by:

$$
\sum_{s \in \Omega} \sum_{i=1}^{L} \frac{B^{i}(s)}{Q^{i}(s)} q_{h}^{i}(s) \geq \sum_{s \in \Omega} \sum_{i=1}^{L} b_{h}^{i}(s)
$$

If such constraint is met, commodities and money are distributed among non bankrupt traders in proportion to their bids and offers, respectively. On the other hand, bankrupt consumers who fail to meet their budget constraint are punished and their purchases are confiscated. Given the strategy profile $(b, q) \in \prod_{h \in H} S_{h}$, the amount of commodity $i, i=1,2, \ldots, L$, consumed by trader $h \in H$ in state $s \in \Omega$ is determined by the following allocation mechanism:

$$
x_{h}^{i}(s ; b, q)=\left\{\begin{array}{lc}
\min _{s^{\prime} \in \Pi_{h}(s)}\left[e_{h}^{i}\left(s^{\prime}\right)-q_{h}^{i}\left(s^{\prime}\right)+b_{h}^{i}\left(s^{\prime}\right) \frac{Q^{i}\left(s^{\prime}\right)}{B^{i}\left(s^{\prime}\right)}\right], & \text { if }(13) \text { holds } \\
\min _{s^{\prime} \in \Pi_{h}(s)}\left[e_{h}^{i}\left(s^{\prime}\right)-q_{h}^{i}\left(s^{\prime}\right)\right], & \text { otherwise }
\end{array}\right.
$$

where $\Pi_{h}(s)$ denotes the block in the partition $\Pi_{h}$ containing the state $s$ and the fraction $\pi^{i}(s ; b, q)=\frac{B^{i}(s)}{Q^{i}(s)}$ can be naturally interpreted as the market clearing 'price' in state s, when $B^{i}(s) Q^{i}(s) \neq 0$.

It can be easily shown that the allocation $x_{h}^{i}(s ; b, q)$ is physically feasible for the asymmetric information economy $\mathcal{E}$; that is, for every state $s \in \Omega$ and for every commodity $i \in\{1, \ldots, L\}$ it holds that:

$$
\sum_{h \in H} x_{h}^{i}(s) \leq \sum_{h \in H} e_{h}^{i}(s) .
$$

It is noteworthy that the allocation mechanism we have adopted considers some form of prudence (see Correia da Silva and Hervés-Beloso, 2009 and De Castro and Yannelis, 2010) by allotting each trader the minimum quantity in every block of his partition. The result is that consumers receive the same bundle in states which they do not distinguish.

\subsection{The strategic market game}

We associate the previous framework with a game $G$, referred to as the strategic market game, where the set of players is $H$, the strategy sets are $\left(S_{h}\right)_{h \in H}$ and the payoffs are given by $\left(P_{h}\right)_{h \in H}$ with $P_{h}$ : $\prod_{h \in H} S_{h} \rightarrow \Re$ defined by $P_{h}(b, q)=u_{h}\left(\left(x_{h}^{i}(s ; b, q)\right)_{i \in\{1, \ldots, L\}, s \in \Omega}\right)$.

A strategy profile $\left(b^{*}, q^{*}\right)$ is a Nash equilibrium for the game $G$ if, for each player $h \in H$,

$$
P_{h}\left(b^{*}, q^{*}\right) \geq P_{h}\left(\left(b_{-h}^{*}, q_{-h}^{*}\right),\left(b_{h}, q_{h}\right)\right), \text { for all }\left(b_{h}, q_{h}\right) \in S_{h}
$$

By following the same argument by Peck et al. (1992), it can be shown that in this model Nash equilibria where agents choose strategies lying in the interior of their strategy sets always exist. They are called fully active Nash equilibria and in the sequel we will focus on such equilibria.

\section{A preliminary result}

In this section we provide a preliminary result which, besides being interesting in its own right, turns to be crucial for the rest of the paper. It states that for every Nash equilibrium $(b, q)$ of the strategic market game $G$, the quantity $e_{h}^{i}-q_{h}^{i}+b_{h}^{i} \frac{Q^{i}}{B^{i}}$ referred to trader $h$, is compatible with his initial information $\Pi_{h}$. 
It is worth noting that in our model, although the allocation mechanism is such that it allots the same quantities in states that traders cannot distinguish, a Nash equilibrium of the game $G$ may potentially involve strategies which vary across these indistinguishable states. We show that each agent strategically chooses bids and offers which guarantee the same commodity bundles in such indistinguishable states of nature. The result has been inspired by a similar one provided by Faias et al. (2011, Lemma 3.1) for a variant of the Shapley and Shubik game introduced by Dubey and Geanakoplos (2003) where a continuum of individuals is involved and every agent $h \in H$ puts up his entire endowment $e_{h}^{i}(s)$ for sale at trading post for commodity $i$ in state $s$.

Proposition 3 Let $(b, q)$ be a fully active Nash equilibrium for the game $G$. Then, for every $h \in H$ and for every $i=1, \ldots, L$, the quantity $e_{h}^{i}-q_{h}^{i}+b_{h}^{i} \frac{Q^{i}}{B^{i}}$ referred to trader $h$ is constant across states of nature that trader $h$ cannot distinguish. That is, for every $s \in \Omega$ :

$$
e_{h}^{i}(s)-q_{h}^{i}(s)+b_{h}^{i}(s) \frac{Q^{i}(s)}{B^{i}(s)}=e_{h}^{i}\left(s^{\prime}\right)-q_{h}^{i}\left(s^{\prime}\right)+b_{h}^{i}\left(s^{\prime}\right) \frac{Q^{i}\left(s^{\prime}\right)}{B^{i}\left(s^{\prime}\right)}, \forall s^{\prime} \in \Pi_{h}(s)
$$

where $\Pi_{h}(s)$ denotes the block of partition $\Pi_{h}$ containing $s$.

\section{Proof .}

By way of contradiction, let $(b, q)$ be a fully active Nash equilibrium such that the measurability condition (14) does not hold. That is, there exist a trader $h \in H$, a commodity $i \in\{1, \ldots, L\}$ and two states of nature $t, \bar{t} \in \Omega$ with $\bar{t} \in \Pi_{h}(t)$ such that:

$$
e_{h}^{i}(t)-q_{h}^{i}(t)+b_{h}^{i}(t) \frac{Q^{i}(t)}{B^{i}(t)} \neq e_{h}^{i}(\bar{t})-q_{h}^{i}(\bar{t})+b_{h}^{i}(\bar{t}) \frac{Q^{i}(\bar{t})}{B^{i}(\bar{t})} .
$$

To keep notation concise, denote for each state $s \in \Omega$ :

$$
\omega_{h}^{i}(s)=e_{h}^{i}(s)-q_{h}^{i}(s)+b_{h}^{i}(s) \frac{Q^{i}(s)}{B^{i}(s)} .
$$

Without loss of generality, assume that $\omega_{h}^{i}(\bar{t})<\omega_{h}^{i}(t)$.

Denote by $\omega^{*}$ the following quantity:

$$
\omega^{*}=\min \left\{\omega_{h}^{i}(s): s \in \Pi_{h}(t)\right\}
$$

and by $S$ the set of states of nature where the minimum is reached, that is:

$$
S=\left\{s \in \Pi_{h}(t): \omega_{h}^{i}(s)=\omega^{*}\right\} .
$$

$S$ is a proper subset of $\Pi_{h}(t)$ since $t \notin S$.

Let $T=\Pi_{h}(t) \backslash S$ and $\omega^{* *}=\min \left\{\omega_{h}^{i}(s): s \in T\right\}$. For the following, it is convenient to index the elements in $T$ and $S$ using natural numbers in such a way that $s_{k} \in T$ iff $k \in\{1, \ldots,|T|\}$ and $s_{k} \in S$ iff $k \in\{|T|+1, \ldots,|S \cup T|\} .{ }^{7}$

Since $T \subset \Pi_{h}(t)$, it holds true that $\omega^{*} \leq \omega^{* *}$. Further, $\omega^{*}<\omega^{* *}$; otherwise, a state $\tilde{s}$ would exist such that $\tilde{s} \in S \bigcap T$, which is impossible.

Denote $\varepsilon=\omega^{* *}-\omega^{*}>0$.

Now, consider the constraint for trader $h$ expressed in terms of the quantity $\omega_{h}^{j}$ :

$$
\sum_{s \in \Omega} \sum_{j=1}^{L} \frac{B_{-h}^{j}(s)\left(\omega_{h}^{j}(s)-e_{h}^{j}(s)\right)}{Q_{-h}^{j}(s)-\omega_{h}^{j}(s)+e_{h}^{j}(s)}=0 .
$$

By regrouping the terms involving commodity $i$ and the states of nature in $\Pi_{h}(t)$, the constraint converts into the following form:

$$
\begin{gathered}
\sum_{s \in \Omega \backslash \Pi_{h}(t)} \sum_{j \neq i} \frac{B_{-h}^{j}(s)\left(\omega_{h}^{j}(s)-e_{h}^{j}(s)\right)}{Q_{-h}^{j}(s)-\omega_{h}^{j}(s)+e_{h}^{j}(s)}+\sum_{k=1}^{|T|} \frac{B_{-h}^{i}\left(s_{k}\right)\left(\omega_{h}^{i}\left(s_{k}\right)-e_{h}^{i}\left(s_{k}\right)\right)}{Q_{-h}^{i}\left(s_{k}\right)-\omega_{h}^{i}\left(s_{k}\right)+e_{h}^{i}\left(s_{k}\right)}+ \\
+\sum_{k=|T|+1}^{|S \cup T|} \frac{B_{-h}^{i}\left(s_{k}\right)\left(\omega_{h}^{i}\left(s_{k}\right)-e_{h}^{i}\left(s_{k}\right)\right)}{Q_{-h}^{i}\left(s_{k}\right)-\omega_{h}^{i}\left(s_{k}\right)+e_{h}^{i}\left(s_{k}\right)}=0 .
\end{gathered}
$$

7 The notation $|T|$ stands for the cardinality of $T$. 
Now denote by $r$ the cardinality of the set $T$ and define the function:

$$
\begin{aligned}
F\left(x_{1}, \ldots, x_{r}, y\right) & =\sum_{s \in \Omega \backslash \Pi_{h}(t)} \sum_{j \neq i} \frac{B_{-h}^{j}(s)\left(\omega_{h}^{j}(s)-e_{h}^{j}(s)\right)}{Q_{-h}^{j}(s)-\omega_{h}^{j}(s)+e_{h}^{j}(s)}+ \\
& +\sum_{k=1}^{r} \frac{B_{-h}^{i}\left(s_{k}\right)\left(x_{k}-e_{h}^{i}\left(s_{k}\right)\right)}{Q_{-h}^{i}\left(s_{k}\right)-x_{k}+e_{h}^{i}\left(s_{k}\right)}+\sum_{k=r+1}^{|S \cup T|} \frac{B_{-h}^{i}\left(s_{k}\right)\left(y-e_{h}^{i}\left(s_{k}\right)\right)}{Q_{-h}^{i}\left(s_{k}\right)-y+e_{h}^{i}\left(s_{k}\right)} .
\end{aligned}
$$

By setting:

$$
y^{0}=\omega^{*}, x_{1}^{0}=\omega_{h}^{i}\left(s_{1}\right), \ldots, x_{r}^{0}=\omega_{h}^{i}\left(s_{r}\right),
$$

it holds that $F\left(x_{1}^{0}, \ldots, x_{r}^{0}, y^{0}\right)=0$ and $F_{y}\left(x_{1}^{0}, \ldots, x_{r}^{0}, y^{0}\right) \neq 0$.

By the implicit function theorem, there exist a neighborhood $N_{\delta} \subset \Re$ of $y_{0}=\omega^{*}$, a neighborhood $N_{\beta} \subset \Re^{r}$ of $\left(\omega_{h}^{i}\left(s_{j}\right)\right)_{j=1}^{r}$ and a function $\varphi: N_{\beta} \rightarrow N_{\delta}, \varphi=\varphi\left(x_{1}, \ldots, x_{r}\right)$, such that:

$$
F\left(x_{1}, \ldots, x_{r}, y\right)=0, \text { for all }\left(x_{1}, \ldots, x_{r}\right) \in N_{\beta} \text { and } y \in N_{\delta} .
$$

Now, define:

$$
\hat{\omega}_{h}^{i}(s)= \begin{cases}\omega_{h}^{i}(s), & \text { if } s \in \Omega \backslash \Pi_{h}(t) ; \\ \omega^{*}+\bar{\delta}, & \text { if } s \in S \\ \omega_{h}^{i}(s)-\bar{\delta}, & \text { if } s \in T\end{cases}
$$

where $\bar{\delta}=\min \left\{\beta, \delta, \frac{\varepsilon}{2}\right\}$.

It holds true that:

$$
F\left(\left(\omega_{h}^{i}(s)-\bar{\delta}\right)_{s \in T}, \omega^{*}+\bar{\delta}\right)=0,
$$

which implies that $\hat{\omega}_{h}^{i}(s)$ satisfies the constraint (15).

In addition, for all $s \in T$ :

$$
\hat{\omega}_{h}^{i}(s)=\omega_{h}^{i}(s)-\bar{\delta} \geq \omega^{* *}-\bar{\delta}=\omega^{*}+\varepsilon-\bar{\delta} \geq \omega^{*}+\frac{\varepsilon}{2} \geq \omega^{*}+\bar{\delta},
$$

which implies:

$$
\min \left\{\hat{\omega}_{h}^{i}(s): s \in \Pi_{h}(t)\right\}=\min \left\{\hat{\omega}_{h}^{i}(s): s \in S\right\}=\omega^{*}+\bar{\delta},
$$

and hence:

$$
\min \left\{\hat{\omega}_{h}^{i}(s): s \in \Pi_{h}(t)\right\}>\min \left\{\omega_{h}^{i}(s): s \in \Pi_{h}(t)\right\} .
$$

This suggests that trader $h$ has an incentive to deviate from the strategy profile $(b, q)$, which is a contradiction to the assumption that $(b, q)$ is a Nash equilibrium.

\section{Results for economies with uncertainty and asymmetric information}

The utility-maximization problem for consumer $h \in H$, which provides his best response to the equilibrium strategies played by all other traders, is the following:

$$
\begin{aligned}
& \max u_{h}\left[\left(\left(x_{h}^{i}(s ; b(s), q(s))\right)_{i=1}^{L}\right)_{s \in \Omega}\right] \\
& \text { such that } \sum_{s \in \Omega} \sum_{i=1}^{L} b_{h}^{i}(s) \leq \sum_{s \in \Omega} \sum_{i=1}^{L} \frac{q_{h}^{i}(s)}{Q^{i}(s)} B^{i}(s)
\end{aligned}
$$

Since utility $u_{h}$ is a concave function of $x_{h}$, the constraint condition in (16) is binding.

By using the notation stated before, that is $\omega_{h}^{i}(s)=e_{h}^{i}(s)-q_{h}^{i}(s)+b_{h}^{i}(s) \frac{Q^{i}(s)}{B^{i}(s)}$, the constraint in (16) can be expressed as follows:

$$
\sum_{s \in \Omega} \sum_{i=1}^{L} \frac{B_{-h}^{i}(s)\left(\omega_{h}^{i}(s)-e_{h}^{i}(s)\right)}{Q_{-h}^{i}(s)-\omega_{h}^{i}(s)+e_{h}^{i}(s)}=0
$$


Bearing in mind that in equilibrium, according to Proposition 3, for every state $s \in \Omega$ and for every $i \in\{1, \ldots, L\}, x_{h}^{i}(s)=x_{h}^{i}\left(s^{\prime}\right)$ for every $s^{\prime} \in \Pi_{h}(s)$, we can express $b_{h}^{i}$ in terms of $x_{h}^{i}$ as follows:

$$
b_{h}^{i}(s)=\frac{B^{i}(s)}{Q^{i}(s)}\left[x_{h}^{i}(s)-e_{h}^{i}(s)+q_{h}^{i}(s)\right],
$$

and hence we obtain the following equalities:

$$
\frac{B^{i}(s)}{Q^{i}(s)}=\frac{B^{i}(s)-b_{h}^{i}(s)}{Q^{i}(s)-\frac{Q^{i}(s)}{B^{i}(s)} b_{h}^{i}(s)}=\frac{B_{-h}^{i}(s)}{Q_{-h}^{i}(s)-x_{h}^{i}(s)+e_{h}^{i}(s)} .
$$

The constraint in (16) can thus be expressed as follows:

$$
\sum_{s \in \Omega} \sum_{i=1}^{L} \frac{B_{-h}^{i}(s)\left(x_{h}^{i}(s)-e_{h}^{i}(s)\right)}{Q_{-h}^{i}(s)-x_{h}^{i}(s)+e_{h}^{i}(s)}=0
$$

and, in light of Proposition 3, the utility maximization problem (16) can be straightforwardly rewritten as

$$
\begin{aligned}
& \max u_{h}\left[\left(\left(x_{h}^{i}(s)\right)_{i=1}^{L}\right)_{s \in \Omega \backslash \sim}\right] \\
& \text { s.t. } \sum_{s \in \Omega} \sum_{i=1}^{L} \sum_{s^{\prime} \in \Pi_{h}(s)} \frac{B_{-h}^{i}\left(s^{\prime}\right)\left(x_{h}^{i}\left(s^{\prime}\right)-e_{h}^{i}(s)\right)}{Q_{-h}^{i}\left(s^{\prime}\right)-x_{h}^{i}\left(s^{\prime}\right)+e_{h}^{i}\left(s^{\prime}\right)}=0
\end{aligned}
$$

where $\Omega \backslash \sim$ is the quotient set of $\Omega$ with respect to the equivalence relation $\sim$ associated with $\Pi_{h}$, that is:

$$
s \sim s^{\prime} \Leftrightarrow s^{\prime} \in \Pi_{h}(s) .
$$

Note that the number of variables reduces from $L \cdot k$ to $L \cdot r$, where $k$ and $r$ denote the total number of states of nature and the number of blocks in partition $\Pi_{h}$, respectively.

Let $\lambda$ denote the Lagrange multiplier associated with the equality constraint and $\Pi_{h}(s)$ be the block in the information partition $\Pi_{h}$ of trader $h$ which contains the state of nature $s$. By writing down the first order conditions for each commodity $i \in\{1, \ldots, L\}$ and for each state $s \in \Omega$ and by summing them over states in the same block of the partition $\Pi_{h}$, we obtain that for every $s \in \Omega$ and for every $i=1, \ldots, L$ :

$$
\sum_{s^{\prime} \in \Pi_{h}(s)} \frac{\partial u_{h}}{\partial x_{h}^{i}(s)}-\lambda \sum_{s^{\prime} \in \Pi_{h}(s)}\left(\pi^{i}\left(s^{\prime}\right)\right)^{2} \frac{Q_{-h}^{i}\left(s^{\prime}\right)}{B_{-h}^{i}\left(s^{\prime}\right)}=0 .
$$

And hence, for all states $s, t \in \Omega$ and for each commodity $i$ :

$$
\frac{\sum_{s^{\prime} \in \Pi_{h}(s)} \frac{\partial u_{h}}{\partial x_{h}^{i}(s)}}{\sum_{t^{\prime} \in \Pi_{h}(t)} \frac{\partial u_{h}}{\partial x_{h}^{i}(t)}}=\frac{\sum_{s^{\prime} \in \Pi_{h}(s)}\left(\pi^{i}\left(s^{\prime}\right)\right)^{2} \frac{Q_{-h}^{i}\left(s^{\prime}\right)}{B_{-h}^{i}\left(s^{\prime}\right)}}{\sum_{t^{\prime} \in \Pi_{h}(t)}\left(\pi^{i}\left(t^{\prime}\right)\right)^{2} \frac{Q_{-h}^{i}\left(t^{\prime}\right)}{B_{-h}^{i}\left(t^{\prime}\right)}} .
$$

By employing the previous equality, we start by providing an estimation for the following quantity, denoted $\gamma_{h}^{i}(x)$ :

$$
\gamma_{h}^{i}(x)=\max _{s, t \in S}\left|\frac{\sum_{s^{\prime} \in \Pi_{h}(s)} \frac{\partial u_{h}}{\partial x_{h}^{i}\left(s^{\prime}\right)}}{\sum_{t^{\prime} \in \Pi_{h}(t)} \frac{\partial u_{h}}{\partial x_{h}^{i}\left(t^{\prime}\right)}} \cdot \frac{\sum_{t^{\prime} \in \Pi_{h}(t)} \pi^{i}\left(t^{\prime}\right)}{\sum_{s^{\prime} \in \Pi_{h}(s)} \pi^{i}\left(s^{\prime}\right)}-1\right|,
$$

which represents a measure of how distant the strategic behavior of individual $h$ is from price taking when just the commodity $i$ is taken into account.

By (20), the previous quantity is equivalent to

$$
\gamma_{h}^{i}(x)=\max _{s, t \in S}\left|\frac{\sum_{s^{\prime} \in \Pi_{h}(s)}\left[\pi^{i}\left(s^{\prime}\right)\right]^{2} \frac{Q_{-h}^{i}\left(s^{\prime}\right)}{B_{-h}^{i}\left(s^{\prime}\right)}}{\sum_{t^{\prime} \in \Pi_{h}(t)}\left[\pi^{i}\left(t^{\prime}\right)\right]^{2} \frac{Q_{-h}^{i}\left(t^{\prime}\right)}{B_{-h}^{i}\left(t^{\prime}\right)}} \cdot \frac{\sum_{t^{\prime} \in \Pi_{h}(t)} \pi^{i}\left(t^{\prime}\right)}{\sum_{s^{\prime} \in \Pi_{h}(s)} \pi^{i}\left(s^{\prime}\right)}-1\right| .
$$


Lemma 4 Let $x^{*}$ be an allocation resulting from a fully active Nash equilibrium for the game $G$. Then, for every individual $h \in H$ and for each commodity $i \in\{1, \ldots, L\}$, it holds true that:

$$
\gamma_{h}^{i}\left(x^{*}\right) \leq \frac{q_{h}^{i}\left(s^{*}\right)}{Q_{-h}^{i}\left(s^{*}\right)}+\frac{b_{h}^{i}\left(t^{*}\right)}{B_{-h}^{i}\left(t^{*}\right)}+\frac{b_{h}^{i}\left(t^{*}\right) q_{h}^{i}\left(s^{*}\right)}{B_{-h}^{i}\left(t^{*}\right) Q_{-h}^{i}\left(s^{*}\right)},
$$

where $\frac{q_{h}^{i}\left(s^{*}\right)}{Q_{-h}^{i}\left(s^{*}\right)}=\min _{s \in \Omega}\left(\frac{q_{h}^{i}(s)}{Q_{-h}^{i}(s)}\right)$ and $\frac{b_{h}^{i}\left(t^{*}\right)}{B_{-h}^{i}\left(t^{*}\right)}=\max _{t \in \Omega}\left(\frac{b_{h}^{i}(t)}{B_{-h}^{i}(t)}\right)$.

Proof.

Let $h \in H$ and $i \in\{1, \ldots, L\}$ be fixed. For every state $t^{\prime} \in \Omega$, it holds that:

$$
\pi^{i}\left(t^{\prime}\right) \frac{B^{i}\left(t^{\prime}\right)}{B_{-h}^{i}\left(t^{\prime}\right)} \geq\left[\pi^{i}\left(t^{\prime}\right)\right]^{2} \frac{Q_{-h}^{i}\left(t^{\prime}\right)}{B_{-h}^{i}\left(t^{\prime}\right)}=\left[\frac{B^{i}\left(t^{\prime}\right)}{Q^{i}\left(t^{\prime}\right)}\right]^{2} \frac{Q_{-h}^{i}\left(t^{\prime}\right)}{B_{-h}^{i}\left(t^{\prime}\right)} \geq \pi^{i}\left(t^{\prime}\right) \frac{Q_{-h}^{i}\left(t^{\prime}\right)}{Q^{i}\left(t^{\prime}\right)}
$$

Hence, by using the previous chain of inequalities, we obtain that for all $s, t \in \Omega$ :

$$
\begin{aligned}
& \frac{\sum_{s^{\prime} \in \Pi_{h}(s)}\left[\pi^{i}\left(s^{\prime}\right)\right]^{2} \frac{Q_{-h}^{i}\left(s^{\prime}\right)}{B_{-h}^{i}\left(s^{\prime}\right)} \sum_{t^{\prime} \in \Pi_{h}(t)} \pi^{i}\left(t^{\prime}\right)}{\sum_{t^{\prime} \in \Pi_{h}(t)}\left[\pi^{i}\left(s^{\prime}\right)\right]^{2} \frac{Q_{-h}^{i}\left(s^{\prime}\right)}{B_{-h}^{i}\left(s^{\prime}\right)} \sum_{s^{\prime} \in \Pi_{h}(s)} \pi^{i}\left(s^{\prime}\right)} \geq \frac{\sum_{s^{\prime} \in \Pi_{h}(s)}\left[\pi^{i}\left(s^{\prime}\right)\right]^{2} \frac{Q_{-h}^{i}\left(s^{\prime}\right)}{\sum^{i}\left(s^{\prime}\right)}}{\sum_{t^{\prime} \in \Pi_{h}(t)}\left[\pi^{i}\left(t^{\prime}\right)\right]^{2} \frac{Q^{i}\left(t^{\prime}\right)}{B_{-h}^{i}\left(t^{\prime}\right)}} \frac{\sum_{t^{\prime} \in \Pi_{h}(t)} \pi^{i}\left(t^{\prime}\right)}{\sum_{s^{\prime} \in \Pi_{h}(s)} \pi^{i}\left(s^{\prime}\right)} \\
= & \frac{\sum_{s^{\prime} \in \Pi_{h}(s)} \pi^{i}\left(s^{\prime}\right) \frac{Q_{-h}^{i}\left(s^{\prime}\right)}{Q^{i}\left(s^{\prime}\right)}}{\sum_{t^{\prime} \in \Pi_{h}(t)} \pi^{i}\left(t^{\prime}\right)} \\
& \sum_{t^{\prime} \in \Pi_{h}(t)} \pi^{i}\left(t^{\prime}\right) \frac{B^{i}\left(t^{\prime}\right)}{B_{-h}^{i}\left(t^{\prime}\right)} \pi_{s^{\prime} \in \Pi_{h}(s)} \pi^{i}\left(s^{\prime}\right) \\
& \min \left\{\frac{Q_{-h}^{i}\left(s^{\prime}\right)}{Q^{i}\left(s^{\prime}\right)}: s^{\prime} \in \Pi_{h}(s)\right\} \sum_{s^{\prime} \in \Pi_{h}(s)} \pi^{i}\left(s^{\prime}\right) \\
= & \frac{B_{-h}^{i}\left(t^{*}\right) Q_{-h}^{i}\left(s^{*}\right)}{B^{i}\left(t^{*}\right) Q^{i}\left(s^{*}\right)}=\frac{\left(B^{i}\left(t^{*}\right)-b_{h}^{i}\left(t^{*}\right)\right)\left(Q^{i}\left(s^{*}\right)-q_{h}^{i}\left(s^{*}\right)\right)}{B^{i}\left(t^{\prime}\right)} \\
\geq & 1-\frac{q_{h}^{i}\left(s^{*}\right)}{B^{i}\left(s^{*}\right)}-\frac{b_{h}^{i}\left(t^{*}\right)}{B^{i}\left(t^{*}\right)}+\frac{b_{h}^{i}\left(t^{*}\right) q_{h}^{i}\left(s^{*}\right)}{B^{i}\left(t^{*}\right) Q^{i}\left(s^{*}\right)} \\
\geq & 1-\frac{q_{h}^{i}\left(s^{*}\right)}{Q_{-h}^{i}\left(s^{*}\right)}-\frac{b_{h}^{i}\left(t^{*}\right)}{B_{-h}^{i}\left(t^{*}\right)}-\frac{b_{h}^{i}\left(t^{*}\right) q_{h}^{i}\left(s^{*}\right)}{B_{-h}^{i}\left(t^{*}\right) Q_{-h}^{i}\left(s^{*}\right)}
\end{aligned}
$$


On the other hand, it also holds that:

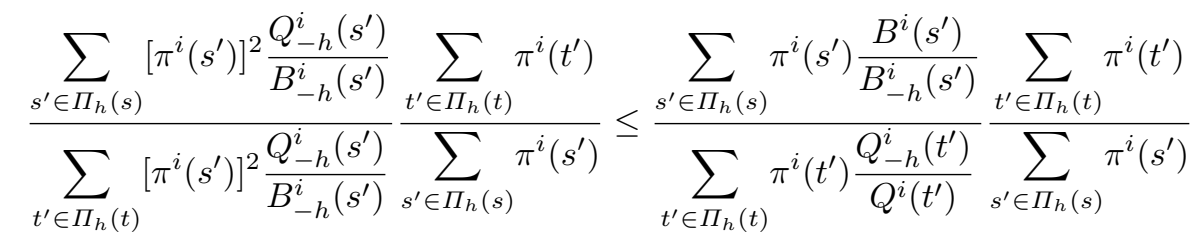

$$
\begin{aligned}
& \leq \frac{\max \left\{\frac{B^{i}\left(s^{\prime}\right)}{B_{-h}^{i}\left(s^{\prime}\right)}: s^{\prime} \in \Pi_{h}(s)\right\} \sum_{s^{\prime} \in \Pi_{h}(s)} \pi^{i}\left(s^{\prime}\right) \sum_{t^{\prime} \in \Pi_{h}(t)} \pi^{i}\left(t^{\prime}\right)}{\sum_{\min }\left\{\frac{Q_{-h}^{i}\left(t^{\prime}\right)}{Q^{i}\left(t^{\prime}\right)}: t^{\prime} \in \Pi_{h}(t)\right\} \sum_{t^{\prime} \in \Pi_{h}(t)} \pi^{i}\left(s^{\prime}\right)} \\
& \leq \frac{\max \left\{\frac{B^{i}\left(s^{\prime}\right)}{B_{-h}^{i}\left(s^{\prime}\right)}: s^{\prime} \in \Omega\right\}}{\min \left\{\frac{Q_{-h}^{i}\left(t^{\prime}\right)}{Q^{i}\left(t^{\prime}\right)}: t^{\prime} \in \Omega\right\}}=\frac{B^{i}\left(t^{*}\right) Q^{i}\left(s^{*}\right)}{B_{-h}^{i}\left(t^{*}\right) Q_{-h}^{i}\left(s^{*}\right)} \\
& =\frac{\left(B_{-h}^{i}\left(t^{*}\right)+b_{h}^{i}\left(t^{*}\right)\right)\left(Q_{-h}^{i}\left(s^{*}\right)+q_{h}^{i}\left(s^{*}\right)\right.}{B_{-h}^{i}\left(t^{*}\right) Q_{-h}^{i}\left(s^{*}\right)} \\
& =1+\frac{q_{h}^{i}\left(s^{*}\right)}{Q_{-h}^{i}\left(s^{*}\right)}+\frac{b_{h}^{i}\left(t^{*}\right)}{B_{-h}^{i}\left(t^{*}\right)}+\frac{b_{h}^{i}\left(t^{*}\right) q_{h}^{i}\left(s^{*}\right)}{B_{-h}^{i}\left(t^{*}\right) Q_{-h}^{i}\left(s^{*}\right)},
\end{aligned}
$$

which concludes the proof.

Starting from $\gamma_{h}^{i}(x)$, consider now, for each trader $h \in H$, the following index $\Gamma_{h}(x)$ which is independent on the commodities:

$$
\Gamma_{h}(x)=\max _{i=1, \ldots, L} \gamma_{h}^{i}(x) .
$$

Next result is an easy consequence of the previous Lemma and its proof is omitted.

Corollary 1 Let $x^{*}$ be an allocation resulting from a fully active Nash equilibrium for the game G. Then, for each individual $h \in H$, it holds true that:

$$
\Gamma_{h}\left(x^{*}\right) \leq \max _{i \in\{1, \ldots, L\}}\left\{\frac{q_{h}^{i}\left(s^{*}\right)}{Q_{-h}^{i}\left(s^{*}\right)}+\frac{b_{h}^{i}\left(t^{*}\right)}{B_{-h}^{i}\left(t^{*}\right)}+\frac{b_{h}^{i}\left(t^{*}\right) q_{h}^{i}\left(s^{*}\right)}{B_{-h}^{i}\left(t^{*}\right) Q_{-h}^{i}\left(s^{*}\right)}\right\} .
$$

We consider now a sequence $\left\{\mathcal{E}_{n}\right\}_{n \in N}$ of economies with asymmetric information and prove that the fraction of traders whose strategic behavior differs from price taking becomes small when the number of traders becomes sufficiently large, independently of the distribution of individual primitives.

Specifically, let $\left\{\mathcal{E}_{n}\right\}_{n \in N}$ be a sequence of economies $\mathcal{E}_{n}: H_{n} \rightarrow \operatorname{Part}(\Omega) \times \Re_{+}^{L \cdot k} \times \mathcal{U}_{c}$, where $\# H_{n} \rightarrow \infty$ and $\lim \frac{1}{\# H_{n}} \sum_{h \in H_{n}} e_{h} \gg 0$. Further, consider the associated sequence of fully active Nash equilibria, $\left(x_{n}\right)_{n \in N}$ with $x_{n} \in \mathcal{N}\left(\mathcal{E}_{n}\right)$ and, for each $n \in N$, let $\left(b_{n}, q_{n}\right) \in \mathbf{N}\left(\mathcal{E}_{n}\right)$ denote the corresponding strategy profile.

The following theorem formally proves what we stated before, thus extending to the asymmetric information framework a result shown in Koutsougeras (2009).

Theorem 3 For each $\epsilon>0$, there is an $n_{\epsilon} \in N$ such that for all $n>n_{\epsilon}$

or, equivalently:

$$
\frac{1}{\# H_{n}} \cdot \#\left\{h \in H_{n}: \Gamma_{h}\left(x_{n}\right)>\epsilon\right\}<\epsilon,
$$

$$
\frac{1}{\# H_{n}} \cdot \#\left\{h \in H_{n}: \Gamma_{h}\left(x_{n}\right)>\epsilon\right\} \rightarrow 0
$$


Proof.

Fix $\epsilon>0$. For $n \in N$, consider the Nash equilibrium $x_{n}$ of the economy $\mathcal{E}_{n}$ and the associated strategy profile $(b, q)^{8}$. For each individual $h \in H_{n}$ let:

$$
\theta_{h, n}=\max _{i \in\{1, \ldots, L\}}\left\{\frac{b_{h}^{i}\left(t_{h}^{*}\right)}{B_{-h}^{i}\left(t_{h}^{*}\right)}, \frac{q_{h}^{i}\left(s_{h}^{*}\right)}{Q_{-h}^{i}\left(s_{h}^{*}\right)}\right\}
$$

where $s_{h}^{*} \in \Omega$ is the state of nature such that $\frac{Q_{-h}^{i}\left(s_{h}^{*}\right)}{Q^{i}\left(s_{h}^{*}\right)}=\min _{s^{\prime} \in \Omega}\left(\frac{Q_{-h}^{i}\left(s^{\prime}\right)}{Q^{i}\left(s^{\prime}\right)}\right)$ and $t_{h}^{*} \in \Omega$ is the state of nature such that $\frac{B_{-h}^{i}\left(t_{h}^{*}\right)}{B^{i}\left(t_{h}^{*}\right)}=\min _{t^{\prime} \in \Omega}\left(\frac{B_{-h}^{i}\left(t^{\prime}\right)}{B^{i}\left(t^{\prime}\right)}\right) 9$.

By Corollary $1, \Gamma_{h}\left(x_{n}\right) \leq\left(\theta_{h, n}\right)^{2}+2 \theta_{h, n}$ and hence:

$$
\begin{aligned}
\#\left\{h \in H_{n}: \Gamma_{h}\left(x_{n}\right)>\varepsilon\right\} & \leq \#\left\{h \in H_{n}:\left(\theta_{h, n}\right)^{2}+2 \theta_{h, n}>\varepsilon\right\} \\
& =\#\left\{h \in H_{n}: \theta_{h, n}>\eta\right\}
\end{aligned}
$$

where $\eta=-1+\sqrt{1+\varepsilon}$.

Define now the following set:

$$
S_{n}=\left\{h \in H_{n}: \frac{b_{h}^{i}\left(t_{h}^{*}\right)}{B_{-h}^{i}\left(t_{h}^{*}\right)}>\eta \text { or } \frac{q_{h}^{i}\left(s_{h}^{*}\right)}{Q_{-h}^{i}\left(s_{h}^{*}\right)}>\eta, \text { for some } i \in\{1, \ldots, L\}\right\} .
$$

It holds that:

$$
\# S_{n} \leq \#\left\{h \in H_{n}: \theta_{h, n}>\eta\right\}
$$

Moreover,

$$
\begin{aligned}
& \frac{b_{h}^{i}\left(t_{h}^{*}\right)}{B_{-h}^{i}\left(t_{h}^{*}\right)}>\eta \Leftrightarrow b_{h}^{i}\left(t_{h}^{*}\right)>B_{-h}^{i}\left(t_{h}^{*}\right) \eta=\left(B^{i}\left(t_{h}^{*}\right)-b_{h}^{i}\left(t_{h}^{*}\right)\right) \eta \Leftrightarrow \\
& \Leftrightarrow b_{h}^{i}\left(t_{h}^{*}\right)(1+\eta)>\eta B^{i}\left(t_{h}^{*}\right) \Leftrightarrow \frac{b_{h}^{i}\left(t_{h}^{*}\right)}{B^{i}\left(t_{h}^{*}\right)}>\frac{\eta}{\eta+1}
\end{aligned}
$$

and, in the same fashion:

$$
\frac{q_{h}^{i}\left(s_{h}^{*}\right)}{Q_{-h}^{i}\left(s_{h}^{*}\right)}>\eta \Leftrightarrow \frac{q_{h}^{i}\left(s_{h}^{*}\right)}{Q^{i}\left(s_{h}^{*}\right)}>\frac{\eta}{\eta+1} .
$$

For each $i \in\{1, \ldots, L\}$, define the following sets:

$$
\begin{aligned}
& T_{i}^{b}=\left\{h \in H_{n}: \frac{b_{h}^{i}\left(t_{h}^{*}\right)}{B_{-h}^{i}\left(t_{h}^{*}\right)}>\frac{\eta}{\eta+1}\right\}, \\
& T_{i}^{q}=\left\{h \in H_{n}: \frac{q_{h}^{i}\left(t_{h}^{*}\right)}{Q_{-h}^{i}\left(t_{h}^{*}\right)}>\frac{\eta}{\eta+1}\right\} .
\end{aligned}
$$

We obtain that:

$$
\# S_{n} \leq \# \bigcup_{i=1}^{L}\left(T_{i}^{b} \cup T_{i}^{q}\right) \leq \sum_{i=1}^{L}\left(\# T_{i}^{b}+\# T_{i}^{q}\right) \leq \frac{2 \cdot k \cdot L \cdot(1+\eta)}{\eta}
$$

8 To keep notation simple, we omit the index $n$ in the strategy profile $(b, q)$.

9 States $s_{h}^{*}$ and $t_{h}^{*}$ vary with $h \in H_{n}$ and notation makes this dependence explicit because it is important for the rest of the proof. 
where $k$ denotes the total number of states of nature and the last inequality is a consequence of the following simple facts:

$$
\begin{aligned}
& k \geq \sum_{h \in T_{i}^{b}} \frac{b_{h}^{i}\left(t_{h}^{*}\right)}{B^{i}\left(t_{h}^{*}\right)} \geq \# T_{1}^{b} \cdot \frac{\eta}{\eta+1} \\
& k \geq \sum_{h \in T_{i}^{q}} \frac{q_{h}^{i}\left(s_{h}^{*}\right)}{Q^{i}\left(s_{h}^{*}\right)} \geq \# T_{1}^{q} \cdot \frac{\eta}{\eta+1} .
\end{aligned}
$$

We can thus conclude that $\#\left\{h \in H_{n}: \Gamma_{h}\left(x_{n}\right)>\epsilon\right\} \leq \frac{2 \cdot k \cdot L \cdot(1+\eta)}{\eta}$. Hence, given any $\epsilon>0$ and $\delta>0$, it is enough to choose $n_{0}$ so that $\# H_{n_{0}}>\frac{2 \cdot k \cdot L \cdot(1+\eta)}{\eta \cdot \delta}$ to conclude the proof.

In view of this theorem, the asymptotic results of the section without uncertainty can be extended mutatis-mutandis to the asymmetric information context.

\section{Concluding remarks}

In this paper we addressed the asymptotic convergence of active Nash equilibria of strategic market games to Walrasian ones for general sequences of economies whose distribution of characteristics has compact support. Both a model with no uncertainty and one with asymmetric information have been considered. Notice that the proof of Theorem 2 provides a rate of convergence which depends on the set of characteristics $T$ (the constants $c$ and $s$ ), but it also depends on the sequence itself (the constant $\xi$, which in turn depends on $\beta$ - the uniform lower bound on offers). This is sensible because in strategic market games there is no parameter $\beta$ that works for all possible sequences: it is possible that some sequences of prices converge to the boundary of the simplex, irrespectively of the set of characteristics. In that case the corresponding sequence of active equilibria converges to one where some markets are inactive, which typically will not be Walrasian. For the same reason a similar qualification on the sequence of Nash equilibria was needed in Proposition 2. Hence, the results in this paper must be understood as asserting that the limit of all sequences of Nash equilibria which remain active in the limit is Walrasian. On the other hand, our results are very general and encompass all similar asymptotic results of this kind. In particular our results require no restrictions on the type of sequences that we consider and most importantly are not confined to the subset of type symmetric Nash equilibria as all other results do. We emphasize however that we do not claim that every Walrasian allocation can be approximated by a sequence of strategic prices and allocations. That is a different issue which requires perhaps further qualifications.

The generality of our approach is evidenced in the way our analysis extends to the asymmetric information context, where we derive an asymptotic analogue of the result in Faias et al. (2011). The assumptions that we use in this case are exactly the same as in the no uncertainty case, except adapted for the new framework. We use exactly the same approach and with a few lemmas, which are necessary for the particular context of asymmetric information, we show that the key results of the no uncertainty case can be readily adapted in the asymmetric information framework. It is noteworthy that the allocation rule that we use (which is inspired by that article), allows us to approximate Radner equilibria as limits of pure strategy Nash equilibria, as opposed to Bayesian Nash equilibria, i.e., we do not impose measurability restrictions on individual strategies. We emphasize that no asymptotic convergence results have been developed before for strategic market games in the asymmetric information context. 


\section{References}

1. Amir, R., S. Sahi, M. Shubik and S. Yao: A strategic market game with complete markets. J. Econ. Theory 51, 126-143 (1990).

2. Anderson, R.: The core in perfectly competitive economies. In: Aumann, R.J., Hart, S. (eds) Handbook of Game Theory, Vol. I, pp. 413-457. Elsevier Science Publishers B.V (1992).

3. Correia da Silva, J. and C. Hervés-Beloso: Prudent expectations equilibrium in economies with uncertainty delivery. Econ. Theory 39, 67-92 (2009).

4. De Castro, L. and N.C. Yannelis: Ambiguity aversion solves the conflict between efficiency and incentive compatibility. Working paper (2010).

5. Dubey, P. and J. Geanakoplos: From Nash to Walras via Shapley-Shubik. J. Math. Econ. 39, 391-400 (2003).

6. Dubey, P. and M. Shubik: A theory of money and financial institutions. The non-cooperative equilibria of a closed economy with market supply and bidding strategies. J. Econ. Theory 17, 1-20 (1978).

7. Faias, M., Hervés-Beloso, C. and E. Moreno-García: Equilibrium price formation in markets with differentially informed agents. Econ. Theory 48, 205-218 (2011).

8. Fugarolas-Alvarez-Ude, G., Hervés-Beloso, C., Moreno-García, E. and J.P. Torres-Martínez: A market game approach to differential information economies. Econ. Theory 38, 321-330 (2009).

9. Giraud, G. and S. Weyers: Strategic market games with a finite horizon and incomplete markets. Econ. Theory 23, 467-491 (2004)

10. Hildenbrand, W.: Core and equilibria of a large economy. Princeton University Press, Princeton, New Jersey (1974).

11. Koutsougeras, L.C.: Convergence of strategic behavior to price taking. Games Econ. Behav. 65, 234-241 (2009).

12. Mas-Colell, A.: The Cournotian foundations of Walrasian equilibrium theory: An exposition of recent theory. In: Hildenbrand, W. (ed) Advances in Economic Theory, pp. 183-224. Cambridge University Press (1982).

13. Peck, J. and K. Shell: On the nonequivalence of the Arrow-Securities game and the contingent-commodities game. In: Barnett,W., Geweke, J., Shell, K., (eds) Economic complexity: Chaos, sunspots, bubbles, and nonlinearity, pp. $61-85$. Cambridge University Press, (1989).

14. Peck, J. and K. Shell: Liquid markets and competition. Games Econ. Behav. 2, 362-377 (1990).

15. Peck, J., Shell, K. and S. Spear: The market game: existence and structure of equilibrium. J. Math. Econ. 21, 271-299 (1992).

16. Postlewaite, A. and D. Schmeidler: Approximate Walrasian equilibria and nearby economies. Int. Econ. Rev. 22, 105-111 (1981).

17. Sahi, S. and S. Yao: The non-cooperative equilibria of a trading economy with complete markets and consistent prices. J. Math. Econ. 18, 325-346 (1989).

18. Shapley, L.S. and M. Shubik: Trade using one commodity as a means of payment. J. Pol. Econ. 85, 937-968 (1977).

19. Shubik, M. and D.P. Tsomocos: A strategic market game with seigniorage costs of Fiat money. Econ. Theory 19, 187-201 (2002)

20. Voliotis, D.: Strategic market games quantal response equilibria. Econ. Theory 27, 475-482 (2006).

21. Yannelis, N.: The core of an economy with differential information. Econ. Theory 1, 183-198 (1991). 